\title{
Photoemission spectra of deuterated silicon clusters: experiment and theory
}

\author{
L. Kronik ${ }^{1, a}$, R. Fromherz ${ }^{2}$, E. Ko ${ }^{1}$, G. Ganteför ${ }^{2}$, and J.R. Chelikowsky ${ }^{1}$ \\ 1 Department of Chemical Engineering and Materials Science, Minnesota Supercomputing Institute, University of Minnesota, \\ Minneapolis, Minnesota 55455, USA \\ 2 Department of Physics, University of Konstanz, 78457 Konstanz, Germany
}

\begin{abstract}
We compare experimentally measured and $a b$ initio computed photoelectron spectra of negatively charged deuterated silicon clusters $\left(\mathrm{Si}_{m} \mathrm{D}_{n}^{-}, 4 \leq m \leq 10,0 \leq n \leq 2\right)$ produced in a plasma environment. Based on this comparison, we discuss the kinetics and thermodynamics of the cluster formation and the effect of deuterium on the geometrical and electronic structure of the clusters.
\end{abstract}

PACS. 36.40.-c Atomic and molecular clusters - 61.46.+w Nanoscale materials: clusters, nanoparticles, nanotubes, and nanocrystals - 73.22.-f Electronic structure of nanoscale materials: clusters, nanoparticles, nanotubes, and nanocrystals

Silicon is undoubtedly the quintessential electronic material [1]. The backbone of the modern electronics industry is, of course, single-crystalline Si. However, many commercial and laboratory devices rely on other forms of silicon - poly-crystalline Si, amorphous $\mathrm{Si}$, porous Si, quantumdot $\mathrm{Si}$, and more. It is therefore natural that much work has been aimed at examining, and perhaps utilizing, the unique properties that Si nano-clusters exhibit. This effort spans over 15 years - from the pioneering experimental and theoretical work of Smalley et al. [2] and Raghavachari et al. [3], respectively, to the present day (Refs. [4-10] are but a few recent examples).

Surfaces of single-crystalline Si are often passivated with hydrogen [11]. Hydrogen is a simple and effective means for such passivation because by bonding to surface $\mathrm{Si}$ atoms it removes dangling bonds, maintains the tetrahedral coordination of Si atoms found in the bulk, and prevents surface dimerization. Consequently, it also removes the surface electronic states typically associated with the bare Si surface. For the same reasons, hydrogen passivation is often used in the preparation of Si quantum dots [12], and surface hydrogen atoms are often added to theoretical calculations involving $\mathrm{Si}$ as a means of suppressing surface effects [13].

In light of the beneficial effects of hydrogen adsorption on macroscopic Si surfaces, it is natural to ask how hydrogen adsorption affects the structural and electronic properties of Si nano-clusters. In this article, we take a

a Present address: Department of Materials and Interfaces, Weizmann Institue of Science, Rehovoth 76100, Israel.

e-mail: leeor.kronik@weizmann.ac.il step towards answering this question, by comparing experimentally measured and $a b$ initio computed results for the photoelectron spectroscopy (PES) of small, negatively charged silicon clusters capped with one or two atoms of a hydrogen isotope - deuterium.

Negatively charged $\mathrm{Si}_{m} \mathrm{D}_{n}$ clusters $(4 \leq m \leq 10$, $0 \leq n \leq 2$, D denoting deuterium), were produced using a pulsed arc cluster ion source $[14,15]$. Bulk silicon was vaporized in a pulsed arc, with the formed hot plasma flushed by $\mathrm{He}$ carrier gas to an extender, into which molecular deuterium was introduced for the generation of deuterated clusters. Clusters were cooled down to approximately room temperature and flushed into vacuum at the extender exit, with no further annealing. Negatively charged clusters were mass-selected in a time-offlight mass spectrometer and their photoelectron spectra were recorded using a "magnetic bottle" time-of-flight electron spectrometer.

All theoretical calculations were based on solving the Kohn-Sham DFT equations within the local density approximation (LDA), using the higher-order finite-difference pseudopotential method [16]. Low-energy metastable isomers of the clusters were obtained via simulated annealing based on Langevin molecular dynamics [17]. Theoretical photoelectron spectra were computed within the constant matrix approximation, i.e., crosssectional effects on the computed density of states (DOS) curves were neglected. The computed PES curves were broadened by averaging the DOS over several picoseconds of isothermal molecular dynamics and then convoluted with a Gaussian to facilitate comparison to experiment. 


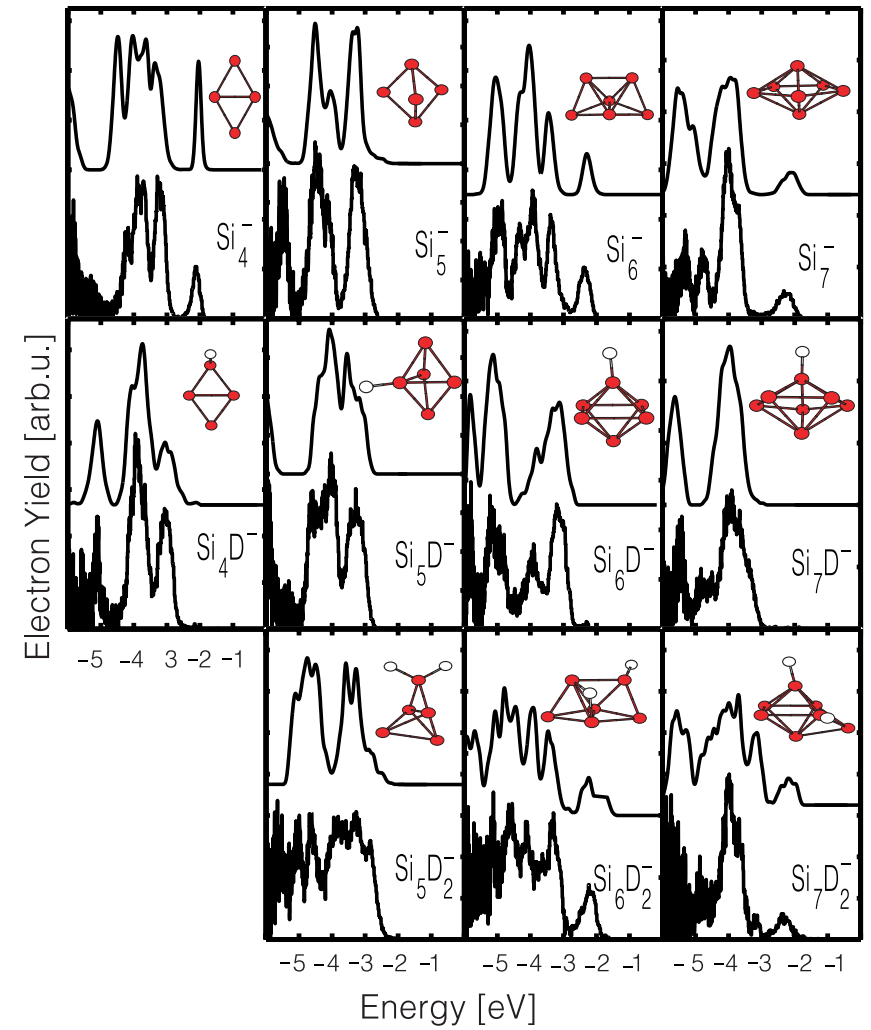

Fig. 1. Experimental PES data (bottom curves), compared with theoretical PES data taken from the isomers yielding best agreement with experiment (top curves), for $\mathrm{Si}_{m} \mathrm{D}_{n}^{-}$clusters $(4 \leq m \leq 7,0 \leq n \leq 2)$. Isomer structures are given as insets.

More complete details of both experiment and theory have been given elsewhere [18].

The experimental and theoretical photoemission spectra of the $\mathrm{Si}_{m} \mathrm{D}_{n}^{-}$clusters, for $4 \leq m \leq 7$ and $8 \leq m \leq 10$ are shown in Figures 1 and 2, respectively. For each cluster type, the theoretical spectrum shown was taken from the isomer shown as an inset, which was the one offering the best agreement between theory and experiment. Clearly, the agreement between theory and experiment is consistently very good to excellent, suggesting that our identification of the observed structures is valid.

Before we proceed to discuss these results, it is important to examine whether experiment probes the ground state isomers or not. If cluster anion formation is kinetically (rather than thermodynamically) limited, minimum total energy considerations alone are not expected to be necessarily predictive of cluster structures. We have previously suggested that under a significant kinetic limitation a highest vertical electron affinity (VEA, the energy gain associated with electron capture by a neutral isomer in the absence of relaxation) would supplant the lowest total energy as the predictive criterion for isomer selection [18]. Briefly, in the hot plasma many neutral isomers can be found with essentially equal probability. Upon charging, high VEA isomers will be favored because electron transfer from a low-VEA isomer to a high-VEA isomer can occur spontaneously, whereas electron transfer in the other

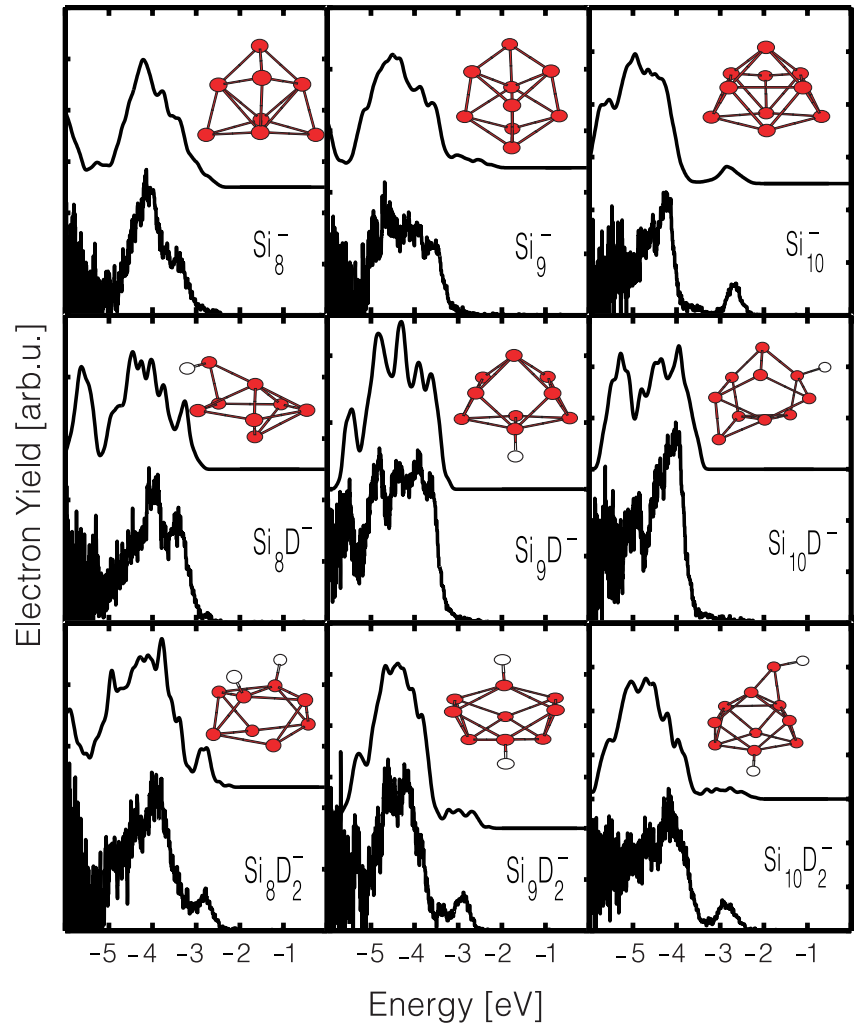

Fig. 2. Experimental PES data (bottom curves), compared with theoretical PES data taken from the isomers yielding best agreement with experiment (top curves), for $\mathrm{Si}_{m} \mathrm{D}_{n}^{-}$clusters $(8 \leq m \leq 10,0 \leq n \leq 2)$. Isomer structures are given as insets.

direction requires energy and is curtailed by fast removal of excess energy via the He carrier gas. If, upon leaving the plasma, the clusters do not have ample time for structural relaxation, they will remain "locked" in their high-VEA, metastable state.

Figure 3 compares, for several cluster types, the experimental PES data, the PES data of the isomer shown in Figure 1, and the PES data for isomers whose spectrum is in worse agreement with theory. Figure 3 also compares the structure, total energy $\left(E_{\text {tot }}\right)$, and VEA of these isomers. Figure $3 \mathrm{a}$, showing the case of $\mathrm{Si}_{8}^{-}$, is clearly an example where the isomer computed to have the lowest energy is in marked disagreement with experiment, whereas the highest VEA isomer agrees very well with experiment. Indeed, for 18 out of the 20 cluster types of Figures 1 and 2, we have found that of the low-energy isomers studied, the highest VEA one provided a spectrum consistent with experiment. In the two other cases (discussed below), we found evidence for partial relaxation of the highest affinity isomer.

The use of the lowest energy criterion can lead to good agreement with experiment for various cluster types. However, there are exceptions, such as the case $\mathrm{Si}_{8}^{-}$mentioned above (Fig. 3a). One possibility is simply that in those irregular cases, the true lowest energy structure was not found by our molecular dynamics run, or that our 


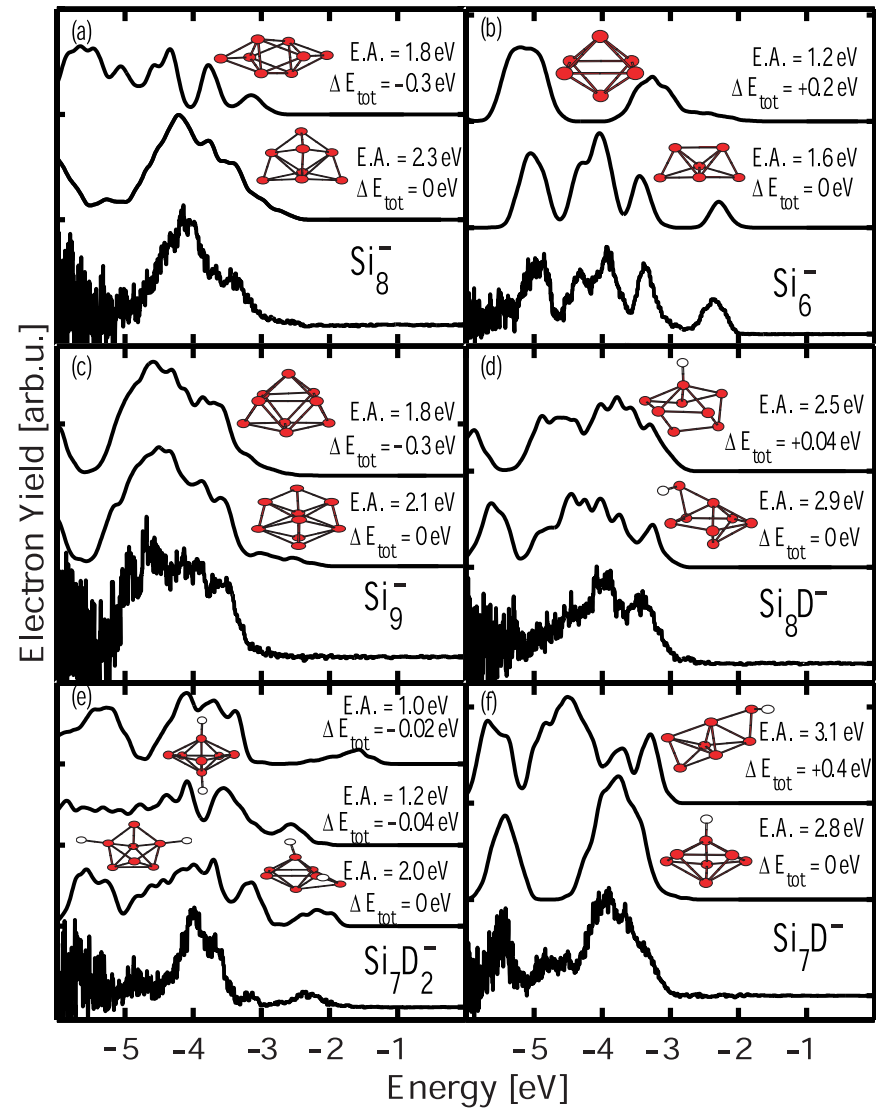

Fig. 3. A comparison of experimental PES data (bottom curves), theoretical PES data based on the isomer yielding best agreement with experiment (middle curves), and theoretical PES data based on different isomers: (a) $\mathrm{Si}_{8}^{-}$, (b) $\mathrm{Si}_{6}^{-}$, (c) $\mathrm{Si}_{9}^{-}$, (d) $\mathrm{Si}_{8} \mathrm{D}^{-}$, (e) $\mathrm{Si}_{7} \mathrm{D}_{2}^{-}$, (f) $\mathrm{Si}_{7} \mathrm{D}^{-}$. Structures, total energy differences (with respect to the isomer yielding best agreement with experiment), and electron affinities for those isomers are given as insets.

computations resulted in an incorrect energy ordering. Indeed, for the bare Si clusters, many of which have been studied previously, different physical approximations and computational details (e.g., choice of basis) have resulted in some variation in energy ordering in the literature $[5$, $8,19]$. We find that our energy ordering for the bare $\mathrm{Si}$ clusters is in consistent agreement with the independent, plane-wave based LDA calculations of Wei et al. [8].

It is also possible, however, that the deviations are not due to computational artifacts, and that low-energy isomers are often favored for other reasons. First, sometimes the lowest energy isomer and the highest VEA isomer are one and the same (e.g., $\mathrm{Si}_{6}^{-}$— see Fig. 3b) [20]. Second, sometimes many structurally different isomers, including the lowest energy and the highest VEA one, lead to practically indistinguishable spectra (e.g., $\mathrm{Si}_{9}^{-}$- see Fig. 3c), so that one cannot tell which is the "observed" isomer. Third, in other cases yet (e.g., $\mathrm{Si}_{8} \mathrm{D}^{-}$— see Fig. 3d), the highest VEA isomer is in somewhat better agreement with experiment than the lowest energy one, but as the discrepancy is not nearly as striking as in, say, Figure 3a, the lowest energy isomer can also be considered to be in general agreement with experiment [21]. For consistency, throughout Figures 1 and 2 we have shown the highest VEA isomer wherever several isomers could be considered to be in agreement with experiment.

Another interesting example is that of $\mathrm{Si}_{7} \mathrm{D}_{2}^{-}$, shown in Figure 3e. We found three essentially quasi-degenerate isomers. Based on energetics alone, all should manifest themselves in the experiment. We fine that the highest affinity one (bottom theoretical spectrum) appears to agree best with experiment. The spectrum of the center isomer is completely devoid of structure below $\sim-4.5 \mathrm{eV}$, in disagreement with experiment. The spectrum of the top isomer is in disagreement with experiment on the position of the high energy peak and completely misses out on the second highest experimental peak.

The only case we found in which the lowest energy isomer was in markedly better agreement with experiment than the highest VEA one was that of $\mathrm{Si}_{7} \mathrm{D}^{-}$, shown in Figure 3f. We believe that this is due to the unusual stability (and therefore, many pathways to relaxation) associated with the bi-capped pentagonal pyramid structure of the isomer [19]. This alleviates the kinetic limitation and relaxation does take place. Still, the experimental feature between $\sim-4.5$ and $\sim-4.8 \mathrm{eV}$ is completely absent in the theoretical PES data corresponding to the ground state structure, but the highest VEA isomer displays a prominent peak at this range. This may be indicative of incomplete relaxation leading to "traces" of the high-VEA isomer in the measured spectrum. In a similar vein, the structure of $\mathrm{Si}_{5} \mathrm{D}_{2}^{-}$shown in Figure 1 corresponds to a structure whose energy is intermediate between the highVEA and the low energy isomers, and also indicates partial relaxation [18].

We now turn to examining some of the trends found in the spectra shown. Generally speaking, in the $\mathrm{Si}_{m} \mathrm{D}_{2}^{-}$ clusters the $\mathrm{D}$ atoms are bound to different silicon atoms, usually on opposite sides of the cluster, indicating significant D-D repulsion. A D-bridge configuration is seen only for $\mathrm{Si}_{6} \mathrm{D}_{2}^{-}$and only in the case of $\mathrm{Si}_{5} \mathrm{D}_{2}^{-}$does Figure 1 show both deuterium atoms to bind to the same silicon atom (neither isomer is the ground state one). Interestingly, with either one or two D atoms, the D atoms do not show a tendency to bind to Si atoms with a small coordination number. The most glaring example is $\mathrm{Si}_{7} \mathrm{D}^{-}$, where the $\mathrm{D}$ atom binds to the cap Si atoms, even though the latter is already bonded to five other Si atoms. This indicates that for clusters this small, the energy loss associated with breaking the highly stable existing structure (in this case the bi-capped pentagonal pyramid) can be larger than the gain associated with increasing the coordination of a single low-coordination Si atom. We therefore conclude that a larger hydrogen coverage would be required before geometries approaching tetrahedral bonding configurations can be expected.

The structural differences between the small deuterated Si clusters and the hydrogenated Si surfaces manifest themselves in the photoemission spectra as well. Generally speaking, the spectra of Figures 1 and 2 become less structured and more broad with increasing number of Si 
atoms. No such trend is observed with the addition of $\mathrm{D}$ atoms, however, and the degree of structure is generally similar to that found for the bare cluster.

Can we observe a phenomenon that would be the equivalent of bulk "passivation" - opening up a bandgap by the removal of surface states? For most of the $\mathrm{Si}_{n}^{-}$and $\mathrm{Si}_{n} \mathrm{D}_{2}^{-}$clusters shown in Figures 1 and 2, a highest occupied molecular orbital (HOMO) - lowest unoccupied molecular orbital (LUMO) gap is clearly observed for both theory and experiment as the separation between the first and second peak in the spectrum. This is because these charged clusters have an odd number of electrons, so that the orbital corresponding to the LUMO in the neutral cluster is half-filled and is probed by photoemission. (For the $\mathrm{Si}_{n} \mathrm{D}^{-}$clusters, the number of electrons is even and the orbital corresponding to the LUMO in the neutral cluster is empty. Consequently, the HOMO-LUMO gap cannot be observed.) Only for the $\mathrm{Si}_{8}$ and the $\mathrm{Si}_{9}$ series do we see that the introduction of two D atoms has resulted in an "opening up of a gap". However, inspection of Figure 3a reveals that the other isomer of $\mathrm{Si}_{8}^{-}$does possess a bandgap even in the absence of deuterium. A "true" opening up of a gap is limited to $\mathrm{Si}_{9}$. This case alone is not enough to determine whether there is already a trend involved (one that would become apparent for $\mathrm{Si}_{11}, \mathrm{Si}_{12}$, etc.) or whether this is an isolated coincidence.

In conclusion, we have compared measured and $a b i n i$ tio computed photoelectron spectra of $\mathrm{Si}_{m} \mathrm{D}_{n}^{-}$clusters produced in a plasma environment. We find evidence for a kinetically limited cluster formation, under which the highest vertical electron affinity is the predictive criterion for cluster structures. By analyzing the trends in structures and spectra, we found that for these small clusters and small deuterium coverage does not induce structures possessing a more tetrahedral bonding structure, and that usually deuterium does not introduce drastic changes in the electronic structure of the cluster. It would be very interesting to examine experimentally and theoretically how and why these conclusions may change for larger hydrogen coverages.

We acknowledge the support provided by the National Science Foundation, the US Department of Energy, the Minnesota Supercomputer Institute, the "Deutsche Forschungsgemeinschaft" and the "Sonderforschungsbereich 513".

\section{References}

1. J.R. Chelikowsky, Mater. Today 5, 64 (2002); J.R. Chelikowsky, MRS Bull. 27, 951 (2003)

2. J.R. Heath, Y. Liu, S.C. O'brien, Q.-L. Zhang, R.F. Curl, F.K. Tittel, R.E. Smalley, J. Chem. Phys. 83, 5520 (1985);
Q.L. Zhang, Y. Liu, R.F. Curl, F.K. Tittel, R.E. Smalley, J. Chem. Phys. 88, 1670 (1988); O. Cheshnovsky, S.H. Yang, C.L. Pettiette, M.J. Craycraft, Y. Liu, R.E. Smalley, Chem. Phys. Lett. 138, 119 (1987)

3. K. Raghavachari, V. Logovinsky, Phys. Rev. Lett. 55, 2853 (1985); K. Raghavachari, J. Chem. Phys. 84, 5672 (1986); K. Raghavachari, C.M. Rohlfing, J. Chem. Phys. 89, 2219 (1988)

4. J. Müller, B. Liu, A.A. Shvartsburg, S. Ogut, J.R. Chelikowsky, K.W.M. Siu, K.-M. Ho, G. Ganteför, Phys. Rev. Lett. 85, 1666 (2000)

5. A.A. Shvartsburg, B. Liu, M.F. Jarrold, K.-M. Ho, J. Chem. Phys. 112, 4517 (2000)

6. L. Mitas, J.C. Grossman, I. Stich, J. Tobik, Phys. Rev. Lett. 84, 1479 (2000)

7. Z.-Y. Li, C.-Z. Wang, K.-M. Ho, Phys. Rev. B 61, 2329 (2000)

8. S. Wei, R.N. Barnett, U. Landman, Phys. Rev. B 55, 7935 (1997)

9. C. Xu, T.R. Taylor, G.R. Burton, D.M. Neumark, J. Chem. Phys. 108, 1395 (1998)

10. K.-M. Ho, A.A. Shvartsburg, B. Pan, Z.-Y. Li, C.-Z. Wang, J.G. Wacker, J.L. Fye, M.F. Jarrold, Nature 392, 582 (1998)

11. A. Many. Y. Goldstein, N.B. Grover, Semiconductor Surfaces, 2nd edn. (North Holland, Amsterdam, 1971)

12. S. Furukawa, T. Miyasato, Phys. Rev. B 38, 5726 (1988)

13. I. Vasiliev, S. Ögüt, J.R. Chelikowsky, Phys. Rev. Lett. 86, $1813(2001)$

14. C.Y. Cha, G. Ganteför, W. Eberhardt, Rev. Sci. Instrum. 63, 5661 (1992)

15. H. Handschuh, G. Ganteför, W. Eberhardt, Rev. Sci. Instrum. 66, 3838 (1995)

16. J.R. Chelikowsky, N. Troullier, Y. Saad, Phys. Rev. Lett. 72, 1240 (1994)

17. N. Binggeli, J.R. Chelikowsky, Phys. Rev. Lett. 75, 493 (1995); J.R. Chelikowsky, N. Binggeli, Mater. Sci. Forum 232, 87 (1996)

18. L. Kronik, R. Fromherz, E. Ko, G. Ganteför, J.R. Chelikowsky, Nature Mat. 1, 49 (2002)

19. K. Raghavachari, C.M. Rohlfing, J. Chem. Phys. 94, 3670 (1991)

20. Interestingly, contrary to this work and to reference [8], Raghavachari and Rohlfing (Ref. [19]) designated the top $\mathrm{Si}_{8}^{-}$and $\mathrm{Si}_{6}^{-}$structures in Figures $3 \mathrm{a}$ and $3 \mathrm{~b}$, respectively, as the ground state ones. Even if their finding is correct, the "observed" isomers would still not correlate with the minimum energy ones, except that $\mathrm{Si}_{6}^{-}$, rather than $\mathrm{Si}_{8}^{-}$, would be the one disobeying the minimum energy rule

21. In fact, due to an over-broadening of the theoretical curve, we have previously [18] shown the lower-affinity isomer as agreeing with experiment 